
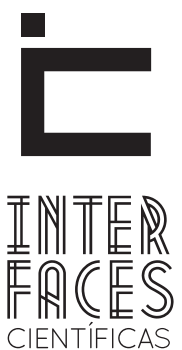

SAÚDE E AMBIENTE

\title{
AS REPRESENTACŌES SOCIAIS DE ADOLESCENTE SOBRE AS DROGAS E IMPLICAÇÕES PARA O CUIDADO DE SI
}

\author{
Silvio Eder Dias da Silva \\ Jeferson Santos do Araújo ${ }^{3}$ \\ Poliana dos Santos Alves ${ }^{5}$ \\ Gilmaira Pires Filgueira?
}

\author{
Esleane Vilela Vasconcelos \\ Jessica Mayra Barboza de Oliveira ${ }^{4}$ \\ Natacha Mariana Farias da Cunha
}

\section{RESUMO}

O estudo objetivou implementar a consulta de enfermagem para adolescentes que vivenciam o consumo de álcool e outras drogas acompanhados pela equipe de enfermagem da Fundação Papa João Paulo II (FUNPAPA) em Belém-PA. Trata-se de um estudo descritivo na modalidade convergente assistencial, no qual aplicou-se a técnica de Livre associação de palavras. As entrevistas foram realizadas com 21 (vinte e um) adolescentes entre 11 (onze) e 19 (dezenove) anos cadastrados e atendidos pelo Projeto Tribos Urbanas em Belém-PA. Empregaram-se os preceitos teóricos da Teoria das Representações Sociais como suporte afim de melhor apresentar o universo que configura o cotidiano desses jovens e adolescentes assistidos. Durante a análise dos dados das entrevistas classificou-se os textos produzidos em quatro categorias temáticas: As Representações Sociais das Drogas como Dor, Sofrimento, e Morte; As Representações Sociais das Drogas como Vestígios da Abstinência; As Representações Sociais das Drogas como algo que faz mal a Saúde e O Cuidado de Si como Cuidado consigo mesmo. Como enfermeiros, cuidadores e promotores da saúde, devemos nos aproximar da realidade de nossos adolescentes a fim de conhecer o problema e contribuir para a elaboração de políticas públicas e programas de prevenção e tratamento para o uso e o abuso de álcool.

\section{PALAVRAS-CHAVE}

Psicologia Social. Cuidados de Enfermagem. Comportamento do Adolescente. 


\section{ABSTRACT}

The study aimed to implement the nursing visits for adolescents who experience the consumption of alcohol and other drugs monitored by the nursing team of the Pope John Paul II Foundation (FUNPAPA) in Belém, PA. This is a descriptive study on the convergent mode, in which we applied the technique of free association of words. Interviews were conducted with 21 (twenty-one) adolescents between eleven (11) and nineteen (19) years registered and serviced by Tribos Urbanas Project in Belém-PA. There were used the theoretical principles of the Theory of Social Representations as support in order to better present the universe that sets the daily lives of these young people and teenagers watched. During the analysis of the interviews data ranked the texts produced in

\section{RESUMEN}

El estudio tuvo como objetivo implementar las consultas de enfermería para los adolescentes que experimentan el consumo de alcohol y otras drogas controladas por el personal de enfermería de la Fundación Juan Pablo II (FUNPAPA) en Belém, PA. Se trata de un estudio descriptivo en el modo convergente, en la que se aplicó la técnica de la asociación libre de palabras. Se entrevistó a 21 (veintiún) adolescentes entre once (11) y diecinueve (19) años registrados y atendidos por Proyecto Tribus Urbanas en Belém-PA. Se emplearon las normas teóricas de la teoría de las representaciones sociales como soporte, para que se obtuviera más éxito a la hora presentar el universo que pone la vida cotidiana de los jóvenes y adolescentes asistidos. Durante el análisis de los datos de las entrevistas se han clasificado textos producidos en cuatro categorí- four thematic categories: Social Representations of Drugs like Pain, Suffering, and Death; Social Representations and Traces of Drugs Abstinence; Social Representations of Drugs as something that makes harm to Health Care and Care of himself/ herself. As nurses, caregivers and health promoters, we must approach the reality of our teenagers to ascertain the problem and contribute to the development of public policies and prevention and treatment programs for the use and abuse of alcohol.

\section{KEYWORDS}

Social Psychology. Nursing Care. Adolescent Behavior.

as temáticas: representaciones sociales de las drogas, tales como las del dolor, sufrimiento y I muerte; las representaciones sociales de las drogas como rastros de abstinencia; representaciones sociales de las drogas como algo que hace daño a la salud, y cuidado del individuo consigo mismo. Como enfermeras, cuidadores y promotores de salud, debemos acercarnos a la realidad de nuestros adolescentes con la finalidad de identificar el problema y contribuir al desarrollo de políticas públicas y programas de prevención y tratamiento respecto al uso y abuso de alcohol.

\section{PALAVRAS CLAVE}

Psicología Social. Cuidados de Enfermería. Conducta del Adolescente. 


\section{INTRODUÇ̄̃O}

A adolescência é considerada uma fase de transição entre a infância e fase adulta, dependendo de circunstâncias sociais e históricas para a formação do individuo. Nesse processo, as experiências vivenciadas, os grupos de amigos, a formação da identidade, valores e descoberta da sexualidade são acontecimentos muito importantes na relação do adolescente com o seu mundo. Pois, é por meio das suas atividades e das suas relações afetivas, principalmente, que o adolescente começa a se definir (MARTINS, 2013).

A adolescência, também, é um período crítico para o desenvolvimento de competências pessoais e interpessoais, aquisição de habilidades para atuação e tomada de decisões. Além de que as vivências de situações de risco tais como delinquência, precocidade nas atividades sexuais, abandono dos estudos, entre outros, constituem formas de comportamento que influenciam no uso da droga, o que acaba sendo uma forma de lidar com as situações problemáticas da vida (SCHENKER; MINAYO, 2014).

O consumo de droga começou a se tornar um problema de saúde pública a partir da década de 1960, devido ao crescente consumo entre jovens, principalmente na adolescência pela vulnerabilidade do ponto de vista psicológico e social e, pelos problemas causados pelo consumo, como os riscos à saúde do usuário e problemas sociais. Dentre os fatores associados ao consumo de drogas, também, estão falta de estrutura familiar, condições socioeconômicas precárias, relacionamento ruim com os familiares (DEZONTINE et al., 2007).

A vista disso, para a compreensão de determinada realidade é necessário identificar a maneira como os sujeitos sociais identificam, explicam e elaboram essa situação e, na adolescência essa realidade ainda está em elaboração (OLIVEIRA et al., 2011). Portanto, as representações sociais vêm com a proposta de designar uma forma de pensamento social, ainda que estas estejam permeadas por co- nhecimentos científicos, mostrando a lógica e as ideias que levam as pessoas a compreender as diferentes ações que realizam (SANCHEZ MARTINEZ; RIBEIRO, 2008).

\section{OBJETIVO}

Implementar a consulta de enfermagem para adolescentes que vivenciam o consumo de álcool e outras drogas acompanhados pela equipe de enfermagem da Fundação Papa João Paulo II (FUNPAPA) em Belém-PA.

\section{MÉTODO}

Trata-se de um estudo descritivo na modalidade convergente assistencial, a qual visa identificar adolescentes e jovens que vivenciam em seu cotidiano o consumo do álcool e de outras drogas. Foram implementadas consultas de enfermagem para a identificação das Representações Sociais dos participantes sobre o presente projeto e como o mesmo implicou na sua avaliação sobre seu autocuidado.

A pesquisa descritiva observa, registra, analisa e correlaciona fatos ou fenômenos (variáveis), sem manipulá-los; estuda fatos e fenômenos do mundo físico e, especialmente, do mundo humano, sem a interferência do pesquisador (GIL, 2010). Trata-se de uma observação não estruturada ou assistemática: consiste em recolher e registrar os fatos da realidade sem que o pesquisador utilize meios técnicos especiais ou precise fazer perguntas diretas (RAMPAZZO, 2012).

A Pesquisa Convergente-Assistencial (PCA) foi desenvolvida por Trentini e Paim e publicada, pela primeira vez, em 1999. A PCA destina-se a ser desenvolvida no mesmo espaço físico e temporal de determinada prática, onde os pesquisadores desenvolvem simultaneamente pesquisa e práticas de saúde com a 
intencionalidade de provocar mudanças qualificadoras daquela assistência. Os instrumentos utilizados serão: um roteiro para a coleta de informações durante a consulta de enfermagem ao adolescente ou jovem atendido pelo programa, e métodos estatísticos para valorar as informações obtidas no decorrer das consultas de enfermagem (TRENTINI; PAIM, 2010).

As informações levantadas na pesquisa serão associadas aos dados obtidos na literatura vigente no banco de dados da Biblioteca Virtual de Saúde, periódicos nacionais e internacionais e livros e revistas que discorrem sobre a temática, respeitando as concepções e práticas de vida individuais e coletivas de saúde, incluindo todo seu universo simbólico.

Serão utilizados preceitos teóricos da Teoria das Representações Sociais como suporte a fim de melhor apresentar o universo que configura o cotidiano desses jovens e adolescentes assistidos. A teoria das representações sociais neste caso reconhece o valor da dimensão subjetiva, o aspecto cognitivo do indivíduo, que segundo esta perspectiva, permite compreender como os mesmos entendem o seu mundo, desde o ambiente das relações sociais até aspectos relativos à própria identidade com o álcool e drogas (MOSCOVICI, 2009).

As entrevistas foram realizadas com 21 (vinte e um) adolescentes entre 11 (onze) e 19 (dezenove) anos cadastrados e atendidos pelo Projeto Tribos Urbanas em Belém - PA, projeto este idealizado pela Fundação Papa João Paulo II (FUNPAPA), que é um programa que atende a adolescentes e jovens envolvidos com bebidas alcoólicas, ou em situações de risco.

A análise de dados será feita por meio da estatística descritiva e do método de analise de conteúdo discutido por Bardin (2008). 0 método da estatística descritiva consiste em um conjunto de técnicas analíticas utilizado para resumir o conjunto de todos os dados coletados numa dada investigação a relativamente poucos números e gráficos.

Para este estudo foram utilizadas a Livre Associação de Palavras, como forma de identificar e analisar as Representações Sociais das drogas nos adolescentes do objeto de estudo. As perguntas utilizadas na Livre Associação de Palavras foram: Quando falo a palavra drogas o que the vem a mente; Quando falo a palavra cuidado de si o que the vem a mente; Quando falo as palavras cuidados com as drogas o que lhe vem a mente

Esta pesquisa está em acordo com o que determina a Resolução 196/96 do Conselho Nacional de Ética em Pesquisa (CONAP) e foi aprovada pelo Comitê de Ética da Universidade Federal do Pará, recebendo o protocolo 004/08 CEP-ICS/UFPA. A pesquisa respeitou os valores culturais, sociais, morais, religiosos e éticos, bem como os hábitos e costumes dos adolescentes. Foi apresentado ao adolescente e/ou ao seu responsável legal um Termo de Consentimento Livre e Esclarecido (TCLE) com linguagem acessível, clara e objetiva.

Os adolescentes que fizeram parte desta pesquisa foram informados da finalidade, justificativa, benefícios, e dos procedimentos que serão utilizados na pesquisa, assim como o desconforto e os benefícios esperados. E ainda, todas as informações e os esclarecimentos que se fizerem necessários serão prestados. O TCLE só será assinado quando houver de ambas as partes a mútua percepção de sua total compreensão.

\section{RESULTADOS}

Durante a análise dos dados das entrevistas emergiram quatro temas que foram: As Representações Sociais das Drogas como Dor, Sofrimento, e Morte; As Representações Sociais das Drogas 
como Vestígios da Abstinência; As Representações Sociais das Drogas como algo que faz mal a Saúde e 0 Cuidado de Si como Cuidado consigo mesmo.

\section{DISCUSSÃO}

\subsection{AS REPRESENTAÇ̃̃ES SOCIAIS DAS DROGAS COMO DOR, SOFRIMENTO, E MORTE}

Atualmente as drogas, além de outros, é grave problema de saúde pública, o pior é que na linha deste perigoso mal se encontram crianças e adolescentes em situações de risco, devido ao consumo de entorpecentes.

Pode-se observar que estes adolescentes têm várias visões quando se pergunta a eles o que lhes vem à mente quando pensam na palavra drogas. Nesta fase da análise observa-se a relação das drogas com dor, sofrimento e morte como nos casos abaixo:

Morte. (E3)

\section{Sofrimento. (E14)}

Morte, desgosto. (E19)

Observa-se também a existência de diálogos mais extensos, relacionados a destruição como:

Vem só coisa que não presta, porque quando uma pessoa usa droga ela fica sem reação, quer fazer tudo, quer tá batendo, tá brigando. (E1)

Destruição. (E17)

Destrói com as famílias do país inteiro. (E18)

Uma coisa que acaba com a família. (E8)

Eles conseguem compreender que as drogas fazem mal para eles, para suas famílias e a dos demais, alguns até se sentem ressentidos pelas atitu- des que as drogas os fazem praticar. Em conversas externas, a parte das perguntas da Livre associação de palavras, os adolescentes costumavam relatar as brigas frequentes com seus familiares, com vizinhos e em alguns casos com colegas de escola. Outros chegaram a relatar, também, a existência de "amigos" que lhes ofereciam as drogas, que poderiam ter acesso à hora que quisessem.

Uma coisa que não presta, que faz mal para gente. (E5)

Maldade, besteira. (E12)

Coisa ruim. (E15)

No momento das entrevistas em alguns casos parei para perguntar: "mas você se lembra do que faz quando está sob o efeito das drogas?", fiz esta indagação porque geralmente o que acontece quando as pessoas tomam bebidas alcoólicas, é o esquecimento ou amnésia temporária, o que em relatos dos mesmos não acontece no caso das drogas, por isso eles dizem que é "algo que não presta" (E5) ou mesmo que vem na mente "Maldade, besteira". (E12). Tive relatos de que o entrevistado se lembrava de tudo, tudo mesmo, inclusive furtos e assaltos cometidos para poder adquirir drogas.

Os adolescentes que fazem uso de drogas geralmente possuem baixa autoestima, às vezes procuram formas de culpar os outros pelos seus problemas ou mesmo fazer o uso das drogas como forma de se integrar e aumentar sua estima. Em vários casos demostram repulsa a si mesmos e aos outros, a autoestima é uma parte do autoconceito. É o juízo pessoal de valor expresso nas atitudes que o indivíduo tem consigo mesmo. É uma experiência subjetiva acessível às pessoas por meio de relatos verbais e comportamentos observáveis, eles também adotam certas atitudes como forma de se destacar, mesmo que de forma negativa (ASSIS et al., 2010). 
A presença positiva ou negativa da autoestima são fatores decisivos na relação do indivíduo consigo mesmo e com os outros, exercendo uma marcante influência na percepção dos acontecimentos e das pessoas, influenciando de forma considerável o comportamento e as vivências do indivíduo (ASSIS et al., 2003).

Por meio disso a Teoria das Representações Sociais reconhece o valor da dimensão subjetiva de cada um, do aspecto cognitivo do indivíduo, que segundo esta perspectiva interfere nas práticas sociais, nas atitudes e condutas relativas ao objeto da representação (SILVA; CAMARGO; PADILHA, 2011a).

\subsection{AS REPRESENTAÇÕES SOCIAIS DAS DROGAS COMO VESTÍGIOS DA ABSTINÊNCIA}

Dentre muitos dos adolescentes pesquisados pode-se observar que eles procuravam encobrir suas verdades quanto a sua relação com as drogas, porém dos 21 pesquisados, sete adolescentes responderam à pergunta: Quando falo a palavra drogas o que lhe vem à mente, com repostas relacionadas ao uso ou lembrança do consumo das drogas. Observamos que a droga ainda está presente em suas vidas no momento em que mostram suas dificuldades:

É que a pessoa não deve usar. Como eu uso eu recomendo a não usar porque não é fácil. (E2)

Vêm várias coisas, vários pensamentos, quando vicia é ruim de sair. (E10)

\section{Nunca usar drogas. (E11)}

Em outros casos a abstinência ficou totalmente nítida, como as vontades de usar novamente e a lembrança do tipo de droga que utilizava como mostram os relatos que seguem:
Penso que vão me oferecer. (E9)

Fumar. (E16)

Pó. (E20)

Fica a fim de fumar. (E21)

Um destes adolescentes que relatou acima, não aceitava a existência das drogas na vida de uma pessoa como dependência química, afirmava não ser dependente, que usava porque queria, na hora que queria e quando desejasse parar pararia, mas o relato é claro, em um adolescente que quando ouve falar a palavra droga a primeira coisa que lhe vem à mente é o nome ou descrição de uma droga, fica evidente a dependência.

Dentro do processo de tratamento e do abandono do uso das drogas, a família tem um papel muito importante, pois na maioria dos casos os adolescentes relataram ter problemas familiares, tais problemas foram diversos, desde a discussão de pai/mãe e filhos como também as discussões entre os pais, sem contar com uso de drogas dentro da própria residência. É importante a inserção da família nos diversos estágios, sendo priorizado o da adolescência, pois é um momento do ciclo vital mais fértil para o uso indevido de drogas (SCHENKER; MINAYO, 2014).

\subsection{AS REPRESENTAÇÕES SOCIAIS DAS DROGAS COMO ALGO QUE FAZ MAL A SAÚDE}

Outros quatro adolescentes fizeram a ligação do consumo das drogas com o fato de realmente fazer mal a saúde, em que demostram sua preocupação e, implicitamente, dão conselhos como segue:

Muitas coisas que acontece. Ela acaba com a saúde. (E4)

Eu sei que é feio, não é bom pros jovens, eu sei que não é bom fazer isso. (E6) 
Uma coisa que não faz bem pra ninguém. (E7)

Uma coisa que faz mal a saúde frequentemente e mexe com a nossa mente. (E13)

A inteligência, a mente, a saúde não só dos adolescentes, mas como de qualquer pessoa que faça o uso de entorpecentes são danificadas; os pensamentos se tornam lentos e vagos, em muitos momentos da entrevista pode-se observar que, em determinados questionamentos, os adolescentes paravam para pensar, mas no mesmo momento não conseguiam fazer o link entre a pergunta e a possível resposta.

A representação social das drogas como algo prejudicial à saúde está no fato de alguns adolescentes não identificarem que, por exemplo, maconha (Cannabis sativa) não seja um tipo de droga, pois em relatos demostram que droga só é aquilo mais pesado ou mais forte como, por exemplo, Cocaína (Erythroxylon coca), Haxixe (é uma variação da Cannabis sativa), Crack (é uma mistura de cocaína em pó, bicarbonato de sódio ou amónia e água destilada) ou Oxi (é pasta base da cocaína, querosene ou gasolina, ácido sulfúrico e cal virgem).

O que se observa é a falta de informação e as situações em que estes adolescentes se encontram vulneráveis. Em casos de revolta alguns adolescentes, na incessante busca de poder e controle sobre si mesmo, de adquirir autonomia e de diferenciar-se de seus pais, escolhem usar drogas, geralmente iniciando com cigarros, álcool, maconha, o que pode levar, consequentemente, ao uso de múltiplas drogas ilícitas, que eles não consideram como sendo tão prejudiciais (WAGNER; OLIVEIRA, 2007).

\subsection{CUIDADO DE SI COMO CUIDADO CONSIGO MESMO}

Em relação ao cuidado de si houve relatos extensos e abrangentes como:

Não sei no momento, é quando agente se cuida assim agente pensa naquilo que tá fazendo, faz bem a nossa saúde. (E13)
É o cara ir ao posto de saúde e ver o que ele tem, se for o caso de remédio compra... (E17)

Cuidar do meu corpo, pra eu não ficar como era antes. (E16)

Estes relatos demonstram a preocupação consigo mesmo, seja em relação às modificações com corpo, seja a ficarem mais propensos a doenças, que habitualmente ocorrem devido ao uso excessivo das drogas, pois se tornam mais relapsos quanto a este assunto, tanto que em relatos que seguem eles demostram de forma mais simples o significado do cuidado de si. Como:

É a pessoa se cuidar. (E2)

Cuidar de mim. (E4)

Cuidar de mim, assim. (E5)

Que eu tenho que me cuidar. (E7)

Cuidar de mim. (E8)

E ter cuidado comigo. (E9)

Sempre cuidar de mim. (E11)

É ter cuidado. (E12)

Em outros relatos os adolescentes responderam com argumentos meio confusos ou desconexos em relação ao indagado.

Me preocupo. (E3)

Pra mim ter cuidado, porque quando agente acha que uma pessoa é tua amiga depois ele vem e mete o pau por trás das tuas costas. (E10)

Me sentir bem. (E15)

Como foi dito anteriormente o uso das drogas influenciaram nas respostas ao questionamento: "Quando falo a palavra cuidado de si, o que the vem a 
mente?", os adolescentes não conseguiam dar respostas mais longas, especificas, que pudessem analisar o que o cuidado consigo representa para eles, o que se observou foi que apesar da indagação e tentativa de respostas por parte dos entrevistadores os adolescentes sempre respondiam de forma curta, generalizada e praticamente uma repetição da pergunta efetuada.

Os adolescentes demostravam não ter muitas informações acerca do cuidado de si, é neste contexto que entra a figura do profissional da saúde, a enfermagem tem um papel decisivo, pois por meio de trabalhos de prevenção e do processo educativo, pode-se evitar o encontro de drogas por pares dos adolescentes, mais especificamente através da educação em saúde. É neste ponto que a educação em saúde pode ser empregada, tanto sob o ponto de vista da prevenção da doença, quanto da promoção da saúde (SILVA; PADILHA; SANTOS, 2011b).

\section{CONCLUSÃO}

A experiência em trabalhar as drogas com os adolescentes foi bastante proveitosa, pois puderam ser identificadas as Representações sociais das drogas na vida dos adolescentes pesquisados. A abordagem foi talvez um pouco confusa para eles, pois por estarem em situação de risco e em alguns casos com problemas judiciários, os mesmos tinham receio em responder o que lhes realmente vinha à mente.

A pesquisa foi bastante árdua, pois ter que indagá-los a ponto de responderem o que realmente perguntávamos foi bem complicado, pois alguns tinham baixa escolaridade, esta prejudicada devido aos hábitos do uso das drogas, e a questão de não compreenderem o indagado.

Na construção do projeto a meta era realizar a entrevista com $90 \%$ dos adolescentes atendidos, que no esperado era de 150 adolescentes. 0 que ocorreu foi que conseguimos alcançar $100 \%$ dos adolescentes atendidos até o momento pelo projeto, com um infeliz detalhe, a taxa de evasão foi intensa e devido a isso o total de adolescentes no momento que constam no projeto é de 21 adolescentes. A alta taxa de evasão deveu-se ao fato de serem cancelados os incentivos aos adolescentes, como incentivo por meio de bolsa por parte de empresas privadas.

Devido à dificuldade de encontrar os adolescentes e de existir a possibilidade deles aceitarem, tivemos alguns casos que eles só aceitaram realizar a entrevista após dois dias de conversa com a Assistente social, com quem eles possuem o vínculo, sendo este de grande valia para a presença dos mesmos.

Como enfermeiros, cuidadores e promotores da saúde, devemos nos aproximar da realidade de nossos adolescentes a fim de conhecer o problema e contribuir para a elaboração de políticas públicas e programas de prevenção e tratamento para o uso e o abuso de álcool, visando sempre à manutenção de uma boa qualidade de vida desses adolescentes longe das drogas.

\section{REFERÊNCIAS}

ASSIS, SG et.al. A representação social do ser adolescente: um passo decisivo na promoção da saúde. Ciência \& Saúde Coletiva. 8(3):669-680, 2003.

BARDIN, L. Análise de conteúdo. Lisboa: Edições 70, 2008.

DEZONTINE, FR et al. Uso de drogas entre adolescentes estudantes da rede privada em São Paulo. ConScietia e Saúde. São Paulo, v.6, n.2, p.323-328, 2007.

GIL, AC. Métodos e técnicas de pesquisa social. 5.ed. São Paulo: Atlas, 2010.

MARTINS, PO et al. 0 ter e o ser: representações sociais da adolescência entre adolescentes de inserção 
urbana e rural. Psicol. Reflex. Crit. Porto Alegre, v.16, n.3, 2013.

MOSCOVICI, S. Representações sociais: investigações em psicologia social. 6.ed. Rio de Janeiro: Vozes, 2009.

OLIVEIRA, DC et al. Futuro e liberdade: o trabalho e a instituição escolar nas representações sociais de adolescentes. Estud. psicol. Natal, v.6, n.2, 2011.

RAMPAZZO, L. Metodologia Científica. 3.ed. São Paulo: Loyola, 2012.

SANCHEZ MARTINEZ, JA; Ribeiro, CRO. Em busca da igualdade: representações do ato de fumar em mulheres adolescentes. Rev. Latino-Am. Enfermagem. Ribeirão Preto, v.16, set-ago, 2008.

SCHENKER, M; MINAYO, MCS. A importância da família no tratamento do uso abusivo de drogas: uma revisão da literatura. Cad. Saúde Pública. Rio de Janeiro, 20(3):649-659, mai-jun, 2004.
SILVA, SED; CAMARGO, BV; PADILHA, MICS. A Teoria das Representações Sociais nas pesquisas da Enfermagem brasileira. Rev Bras Enferm. Brasília, set-out; 64(5):947-51, 2011 (a).

SILVA, SED; PADILHA, MICS; SANTOS, LMS. A enfermagem estimulando o autocuidado de adolescentes a partir das representações sociais desses sobre as bebidas alcoólicas. Enfermagem em Foco. 2(3):160163, 2011 (b).

TRENTINI, M; Paim, L. Pesquisa em enfermagem: uma modalidade convergente-assistencial. Florianópolis: UFSC, 2010.

WAGNER, MF, OLIVEIRA, MS. Habilidades Sociais e Abuso de Drogas em Adolescentes. Psic. Clin., v.19, n.2, p.101-116, Rio de Janeiro, 2007. 
1. Orientador e professor da Universidade Federal do Pará. Doutor em Enfermagem pelo DINTER UFPA/UFSC Belém (PA) Brasil. E-mail: silvioeder@ufpa.br

2. Professora da Universidade Federal do Pará. Mestre em Enfermagem pela Universidade do Estado do Pará. Belém (PA) Brasil. E-mail: leanevas@hotmail.com

3. Doutorando do Doutorado da USP/RP. E-mail: jeferson-ma@ig.com.br

4. Enfermeira formada pela Faculdade de Enfermagem da UFPa. E-mail: jessibarboza@gmail.com.

5. Discente de graduação em Enfermagem da Universidade Federal do Pará - Bolsista PIBIC/CNPq. Membro do Grupo de Pesquisa Educação, Políticas e Tecnologia em Enfermagem da Amazônia (EPOTENA). Belém (PA) Brasil. E-mail: polianaalves_@hotmail.com

6. Discente de graduação em Enfermagem da Universidade Federal do Pará - Bolsista PIBIC/CNPq. Membro do Grupo de Pesquisa Educação, Políticas e Tecnologia em Enfermagem da Amazônia (EPOTENA). Belém (PA) Brasil.

7. Discente de graduação em Enfermagem da Universidade Federal do Pará. Belém (PA) Brasil. E-mail: gilmaira_enfermagem@hotmail.com 